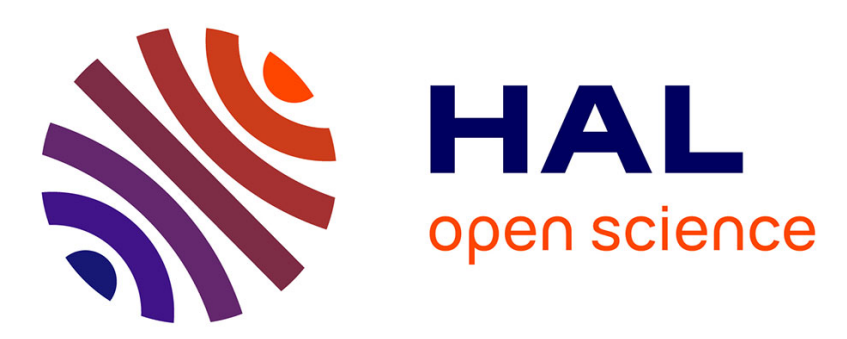

\title{
A moving mesh approach for the numerical simulation of gas micro flows
}

Guillaume Dechristé, Luc Mieussens

\section{To cite this version:}

Guillaume Dechristé, Luc Mieussens. A moving mesh approach for the numerical simulation of gas micro flows. 28TH INTERNATIONAL SYMPOSIUM ON RAREFIED GAS DYNAMICS, Jul 2012, Zaragoza, Spain. pp.366-372, 10.1063/1.4769544 . hal-01036590

\section{HAL Id: hal-01036590 \\ https://hal.science/hal-01036590}

Submitted on 22 Jul 2014

HAL is a multi-disciplinary open access archive for the deposit and dissemination of scientific research documents, whether they are published or not. The documents may come from teaching and research institutions in France or abroad, or from public or private research centers.
L'archive ouverte pluridisciplinaire HAL, est destinée au dépôt et à la diffusion de documents scientifiques de niveau recherche, publiés ou non, émanant des établissements d'enseignement et de recherche français ou étrangers, des laboratoires publics ou privés. 


\title{
A Moving Mesh Approach for the Numerical Simulation of Gas Micro Flows
}

\author{
G. Dechristé and L. Mieussens \\ Univ. Bordeaux, IMB, UMR 5251, F-33400 Talence, France. CNRS, IMB, UMR 5251, F-33400 Talence, France.
}

\begin{abstract}
The study of numerical methods for the computation of gas micro flows in domains with moving obstacles is motivated by the modelling of micro-electromechanical system (MEMS), especially vibrating devices. In this framework, the flow is assumed to be well described by the BGK equation. We proposed a new numerical method for the simulation of deterministic rarefied flows with moving obstacles based on a moving mesh approach.
\end{abstract}

Keywords: Boltzman equation, moving mesh methods, flow simulations

PACS: 47.45.-n, 47.11.Df, 47.61.Fg

\section{INTRODUCTION}

In the framework of rarefied gas flows, several phenomena are observed and do not exist when the gas is described by the classical continuous gas dynamics such that Navier-stokes equations. The gas needs then to be treated at microscopic level by a statistical approach. At this scale the gas is governed by the Boltzmann equation [1]. This kinetic description is justified when the mean free path of molecules is the same order of magnitude that the typical flow dimension.

Rarefied regimes may appears at atmospheric pressure if the typical size of the system is the order of the nano or micrometer. This is precisely the case in MEMS (Micro-Electro-Mechanical-System) [2]. However, rarefied flows also appears in larger system when the ambient pressure is around $10^{-2}$ millibar, for instance in specific pumps. Both systems may be made up of moving parts and the simulation of these systems is still challenging.

For now, several methods for the computation of continuous gas dynamics equation have been proposed. These methods are divided in two main categories: either the computational mesh does not change during the computation, or the grid evolves in order to fit with the physical boundary at each time. Methods based on non-moving mesh are called immersed boundary methods and have been first proposed by Peskin [3] to study blood flow in vessel. An extension of this approach to rarefied gas flows is described in [4]. In this article we proposed to use the moving mesh method [5] for the computation of the simplified BGK model of Boltzmann equation.

The outline of this article is the following. In the first section, we briefly present the BGK model of Boltzmann equation. In the next section, the moving mesh method is described. Finally, in the last section the method is validated on two different test cases: a moving piston and an actuator.

\section{THE BGK MODEL}

The BGK model was proposed by Bhatnagar, Gross and Krook [6] as a simplification of the Boltzmann equation. It consists of a relaxation of the distribution function towards its corresponding Maxwellian equilibrium. In this article, only one dimensional cases in space and velocity have been studied. We empathize that this simplification is non relevant from a physical point of view.

$$
\frac{\partial f}{\partial t}+v \cdot \frac{\partial f}{\partial x}=\frac{1}{\tau}(\mathscr{M}[f]-f) .
$$

The relaxation time $\tau$ is defined by $\tau=\mu /(\rho R T)$, and according to [7], $\mu=\mu_{r e f}\left(T / T_{r e f}\right)^{\omega}$ where $\mu_{r e f}, \omega$ and $T_{r e f}$ are constants that depend on the gas. Because the distribution function $f$ is the density probability of finding a particle at coordinate $x$ with a velocity $v$, the usual macroscopic quantities are its first moments:

$$
(\rho, \rho u, E)=\int_{\mathbb{R}}\left(1, v, \frac{1}{2}|v|^{2}\right) f(t, x, v) d v,
$$


The temperature $T$ of the gas is deduced from the relation $E=\frac{1}{2} \rho|u|^{2}+\frac{1}{2} \rho R T$ where $R$ is the gas constant, and is defined by the ratio between the Boltzmann constant and the molecular mass of the gas. The Maxwellian distribution, which corresponds to the thermodynamical equilibrium state, is obtained from the moments of $f$ :

$$
\mathscr{M}[f]=\frac{\rho}{\sqrt{2 \pi R T}} \exp \left(-\frac{|v-u|^{2}}{2 R T}\right) .
$$

Boundary conditions may be modelled by two standard approaches: either specular or diffuse reflection. In the former model, incident molecules of velocity $v$ are remitted with a velocity symmetric to $v$ with respect to the wall. Consequently, if the wall moves at a velocity $u_{w}$, the specular reflection condition will be given by the following relation:

$$
f(t, x, v)=f\left(t, x, 2 u_{w}-v\right) \quad \text { if } \quad\left(v-u_{w}\right) \cdot n_{x}>0,
$$

where $n_{x}$ is the unit normal vector to the boundary, pointed to the gas. In the diffuse reflection model, the molecules colliding with the boundary are remitted with a temperature $T_{w}$ equal to the wall temperature and with a random velocity normally distributed around $u_{w}$. This reads:

$$
\begin{gathered}
f(t, x, v)=\phi \mathscr{M}\left(1, u_{w}, T_{w}\right) \quad \text { if } \quad\left(v-u_{w}\right) \cdot n_{x}>0, \\
\phi=-\frac{\int_{\left(v-u_{w}\right) \cdot n_{x}<0} f(t, x, v)\left(v-u_{w}\right) \cdot n_{x} d v}{\int_{\left(v-u_{w}\right) \cdot n_{x}>0} \mathscr{M}\left(1, u_{w}, T_{w}\right)\left(v-u_{w}\right) \cdot n_{x} d v} .
\end{gathered}
$$

Note that the coefficient $\phi$ is chosen in order to set the normal mass flux across the wall to zero.

Conservations laws for the density, momentum and energy are obtained by the definition of the moments of $f$. Indeed:

$$
\frac{\partial}{\partial t}\left[\int_{\mathbb{R}}\left(1, v, \frac{1}{2}|v|^{2}\right) \cdot f\right]+\frac{\partial}{\partial x}\left[\int_{\mathbb{R}}\left(v, v^{2}, \frac{1}{2}|v|^{2} v\right) \cdot f\right]=0,
$$

and using Eq. (2), relation (6) become:

$$
\begin{aligned}
\frac{\partial \rho}{\partial t}+\frac{\partial \rho u}{\partial x} & =0, \\
\frac{\partial \rho u}{\partial t}+\frac{\partial\left[\rho|u|^{2}+\rho R T\right]}{\partial x} & =0, \\
\frac{\partial E}{\partial t}+\frac{\partial\left[u\left(\frac{1}{2} \rho|u|^{2}+\frac{3}{2} \rho R T\right)+q\right]}{\partial x} & =0 .
\end{aligned}
$$

The calculation of (7c) is detailed in [1] and the heat flux $q$ is given by the relation $q=\frac{1}{2} \int_{\mathbb{R}}(v-u)^{3} f$. This term may be approximated by a Chapman-Enskog expansion [8], and we get:

$$
q=\frac{3}{2} \tau \rho R^{2} T \frac{\partial T}{\partial x}
$$

For small Knudsen numbers, that is $\tau \rightarrow 0$, the heat flux $q$ is negligible and the set of Eq. (7) is simplified to Euler equations. Otherwise, the set of Eq. (7) is exactly the Navier-Stokes equations. It is generally admitted [7] that the macroscopic equations are no longer sufficient when the Knudsen number is greater than 0.1 .

\section{MOVING MESH APPROACH}

\section{BGK Equation in a Moving Frame}

Let $x_{\min }(t)$ and $x_{\max }(t)$ be the coordinates of the moving frame boundaries. Let also $u_{\min }$ and $u_{\max }$ stand for the velocity of these boundaries. The BGK equation may now be written as:

$$
\begin{aligned}
& \frac{\partial f}{\partial t}+v \cdot \frac{\partial f}{\partial x}=\frac{1}{\tau}(\mathscr{M}[f]-f), \\
& f\left(x_{\text {min }}(t), v, t\right)=f_{\text {min }}\left(x_{\text {min }}(t), v, t\right) \quad \forall\left(v-u_{\text {min }}\right)>0, \\
& f\left(x_{\text {max }}(t), v, t\right)=f_{\text {max }}\left(x_{\text {max }}(t), v, t\right) \quad \forall\left(v-u_{\text {max }}\right)<0 .
\end{aligned}
$$


The boundary conditions $f_{\min }$ and $f_{\max }$ are either specular (4) or diffuse (5). Let us derive the Eq. (9) in a moving frame. Denoting by $u(x, t)$ the velocity of the frame, we consider the following differential system:

$$
\left\{\begin{array}{l}
u(x, t)=\frac{d x}{d t}, \\
x(0)=\xi
\end{array}\right.
$$

The solution of this system is denoted by $x(\xi, t)$. Then $(\xi, t)$ are called the the moving frame coordinates. Moreover, setting:

$$
J(\xi, t)=\left.\frac{\partial x}{\partial \xi}\right|_{\xi, t},
$$

we get:

$$
\begin{aligned}
\left.\frac{\partial J}{\partial t}\right|_{\xi, t} & =\left.\frac{\partial}{\partial t} \frac{\partial x}{\partial \xi}\right|_{\xi, t}=\left.\frac{\partial}{\partial \xi} \frac{\partial x}{\partial t}\right|_{\xi, t}=\left.\frac{\partial u(x, t)}{\partial \xi}\right|_{\xi, t} \\
& =\left.J(\xi, t) \cdot \frac{\partial u}{\partial x}\right|_{x(\xi, t), t} .
\end{aligned}
$$

From now on, for all functions $\Psi(x, t)$ expressed in the usual coordinates, we introduce the notation $\bar{\Psi}(\xi, t)=\Psi(x(\xi, t), t)$. Here, $\bar{\Psi}$ is the function expressed in the moving frame coordinates. Then, using relations (10) and (12), we may compute Eq. (9a) in the moving frame coordinates:

$$
\begin{aligned}
\frac{\partial}{\partial t}(J \bar{f}) & =\bar{f} \cdot \frac{\partial J}{\partial t}+J \cdot \frac{\partial \bar{f}}{\partial t}=J \times\left[\bar{f} \cdot \frac{\overline{\partial u}}{\partial x}+\frac{\overline{\partial f}}{\partial t}+\bar{u} \cdot \overline{\partial_{x} f}\right] \\
& =J \times\left[\frac{\partial f}{\partial t}+\frac{\partial f u}{\partial x}\right] .
\end{aligned}
$$

Noticing that

$$
J \cdot \frac{\overline{\partial f}}{\partial x}=\frac{\partial \bar{f}}{\partial \xi},
$$

and using equality (9a), we can simplify Eq. (13):

$$
\begin{array}{rr}
\frac{\partial}{\partial t}(J \bar{f})+\frac{\partial}{\partial \xi}(\overline{f(v-u)})=J \cdot \frac{1}{\tau}(\mathscr{M}[\bar{f}]-\bar{f}) \\
\bar{f}\left(\xi_{\text {min }}, v, t\right)=f_{\text {min }}\left(\xi_{\text {min }}, v, t\right) & \forall\left(v-u_{\text {min }}\right)>0, \\
\bar{f}\left(\xi_{\text {max }}, v, t\right)=f_{\text {max }}\left(\xi_{\text {max }}, v, t\right) & \forall\left(v-u_{\text {max }}\right)<0 .
\end{array}
$$

\section{Numerical Discretization}

The computational grid is changing at each time step. At each time step $t^{n}=\Delta t$, we denote $x_{\min }^{n}$ and $x_{\text {max }}^{n}$ the value of $x_{\min }\left(t^{n}\right)$ and $x_{\max }\left(t^{n}\right)$. Then, the domain is discretized by a Cartesian grid of $N+1$ nodes $x_{i+1 / 2}^{n}=x_{\min }^{n}+i \Delta x^{n}$ where $\Delta x^{n}=\left(x_{\max }^{n}-x_{\min }^{n}\right) / N$. At initial state, we have:

$$
x_{i}^{0}=\xi_{i} \quad \text { and } \quad \Delta x^{0}=\Delta \xi .
$$

With this mesh definition, the number of cell is the same in each time step, and the space step is constant for a specific time, see Fig. 1. Let now $f_{i}^{n}(v)$ stands for the average of $f\left(x, v, t^{n}\right)$ over a cell $C_{i}^{n}=\left[x_{i-1 / 2}^{n}, x_{i+1 / 2}^{n}[\right.$. Similarly, we denote by $\bar{f}_{i}^{n}(v)$ the average of $\bar{f}\left(\xi, v, t^{n}\right)$ over a cell $\bar{C}_{i}=\left[\xi_{i-1 / 2}, \xi_{i+1 / 2}\right.$. Eq. (11) is approximated by

$$
J\left(\xi, t^{n}\right)=\frac{\Delta x^{n}}{\Delta \xi} \quad \forall \xi \in\left[\xi_{i-1 / 2}, \xi_{i+1 / 2}[\right.
$$




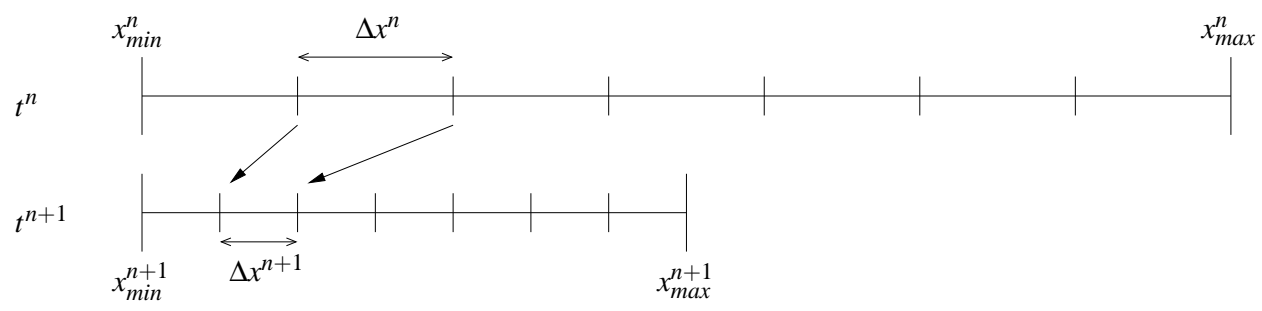

FIGURE 1. Example of grid and space step computation.

and an easy computation shows that $f_{i}^{n}(v)=\bar{f}_{i}^{n}(v)$ :

$$
f_{i}^{n}(v)=\frac{1}{\Delta x^{n}} \int_{x_{i-1 / 2}^{n}}^{x_{i+1 / 2}^{n}} f\left(x, v, t^{n}\right) d x=\frac{1}{\Delta x^{n}} \int_{\xi_{i-1 / 2}}^{\xi_{i+1 / 2}} \bar{f}\left(\xi, v, t^{n}\right) J\left(\xi, t^{n}\right) d \xi=\bar{f}_{i}^{n}(v) .
$$

A discrete velocity method [9] is applied to treat the velocity variable. We define $f_{i, k}^{n}$ as an approximation of $f_{i}^{n}\left(v_{k}\right)$ where $v_{k}$ are the nodes of a bounded Cartesian grid with step $\Delta v$. The Maxwellian is approximated on this grid by a discrete Maxwellian which has the same moments as $f$. Denoting $\mathscr{F}_{i+\frac{1}{2}, k}^{n}$ the time average of $\left[v_{k}-\right.$ $\left.u\left(x_{i+1 / 2}, t\right)\right] f\left(\xi_{i+1 / 2}, v_{k}, t\right)$ between $t$ and $t+\Delta t$, Eq. (15a) is discretized as follows:

$$
\frac{1}{\Delta t}\left(\frac{\Delta x^{n+1}}{\Delta \xi} f_{i, k}^{n+1}-\frac{\Delta x^{n}}{\Delta \xi} f_{i, k}^{n}\right)+\frac{1}{\Delta \xi}\left(\mathscr{F}_{i+\frac{1}{2}, k}^{n}-\mathscr{F}_{i-\frac{1}{2}, k}^{n}\right)=\frac{1}{\tau_{i}^{n}}\left(\mathscr{M}_{i, k}^{n}-f_{i, k}^{n}\right) .
$$

Under the assumption that the velocities of the grid points are constant over a time step $\Delta t$, we denote $u_{i+1 / 2}^{n}=u\left(x_{i+1 / 2}, t\right)=\left(x_{i+1 / 2}^{n+1}-x_{i+1 / 2}^{n}\right) / \Delta t$. The fluxes at the interfaces between two cells can then be computed by a classical first order upwind scheme, and relation (19) is finally simplified to:

$$
\begin{array}{r}
f_{i, k}^{n+1}=\frac{\Delta x^{n}}{\Delta x^{n+1}} f_{i, k}^{n}-\frac{\Delta t}{\Delta x^{n+1}}\left(\mathscr{F}_{i+\frac{1}{2}, k}^{n}-\mathscr{F}_{i-\frac{1}{2}, k}^{n}\right)-\frac{\Delta x^{n}}{\Delta x^{n+1}} \frac{\Delta t}{\tau_{i}^{n}}\left(\mathscr{M}_{i, k}^{n}-f_{i, k}^{n}\right), \\
\mathscr{F}_{i+\frac{1}{2}}^{n}=\max \left(0, v_{k}-u_{i+1 / 2}^{n}\right) \cdot f_{i, k}^{n}+\min \left(0, v_{k}-u_{i+1 / 2}^{n}\right) \cdot f_{i+1, k}^{n} .
\end{array}
$$

Note that with this scheme, the computation of the flux (20b) at the left of the domain (resp. right) requires the value of $f_{0, k}^{n}$ (resp. $f_{N+1, k}^{n}$ ) for all positive (resp. negative) microscopic velocity $v_{k}-u_{\text {min }}^{n}$ (resp. $v_{k}-u_{\max }^{n}$ ). These values are defined by the boundary condition (15b) (resp. (15c) ). Since the velocities of the grid points are related to the speeds of the boundaries, the computation of the time step is linked to the motion of the wall through the CFL condition:

$$
\Delta t<\min \left(\frac{1}{\tau_{i}}, \frac{\Delta x^{n+1}}{v_{k}-u_{i+1 / 2}^{n}}\right)
$$

It is worth pointing out that the positivity and stability properties of the scheme hold in a unbounded domain under this CFL condition. This scheme is order one in space and time. The total order of the scheme can be increased using for instance a Runge-Kutta 2 scheme for temporal discretization and computing the numerical fluxes with a superbee scheme [10]. Equation (15a) can also be treated semi-implicitly [11], so that the CFL condition (21) does not depend on $\tau$.

\section{NUMERICAL RESULTS}

\section{Oscillating Piston}

Russo and Filbet recently proposed a semilagrangian scheme [12] for the numerical simulation of one dimensional rarefied gas flows with moving boundaries. In this section, the same test case that in [12] is studied. The gas is located in 
a one dimensional slab and is governed by a dimensionless BGK equation: $R=1$ and $\tau$ is set to $10^{-3}$. A moving piston is located at the left edge of the slab and moves with a velocity $u_{\min }(t)=0.25 \sin (t)$. Its initial position is $x_{\min }(0)=1$. There is a fixed wall at the right side of the slab located at $x_{\max }=20$. Each edge of the domain is modelled with specular boundary conditions (4). The initial state of the gas is given by $(\rho, u, T)=(1,0,3.15)$. Since the relaxation time $\tau$ is very small, the solutions of the BGK equation can be compared to the solution of Euler equations. An easy way to solve Euler equations in a one dimensional moving frame is to write them in Lagrangian coordinates [13]. Computations are done from $t=0$ to $t=35$. The pressure at each edge of the slab is represented Fig. 2, and results of Euler equations are compared to results obtained with BGK equation. In order to get a very accurate pressure distribution, 2000 cells were used for the computation of Euler equations. The BGK equation has been computed with the moving mesh method (first order scheme) as well as with the semilagrangian scheme. Both computations have been launched with 300 cells. In this case, increasing the number of computational cells or increasing the order of the scheme would lead to better agreements with results of Euler computations. Nevertheless, even with a low space resolution, BGK equation solved with the moving mesh method gives quite accurate results.
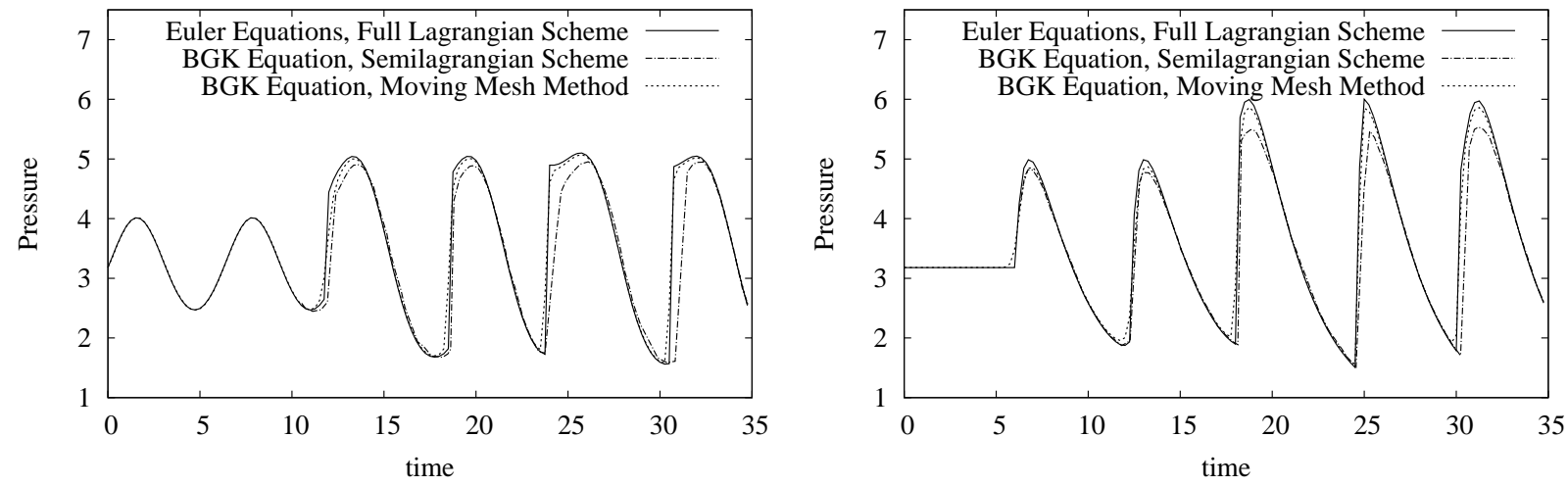

FIGURE 2. Comparisons between the pressure profile obtained with Euler equations and BGK model near the piston (left) and near the fixed wall (right). BGK equation is solved with both semilagrangian and moving mesh method.

\section{Actuator Simulation}

The system is made up of a moving plate located in the center of a slab. The considered gas is argon (molecular mass $\left.M=66.3 \cdot 10^{-27} \mathrm{~g}\right)$ and it is governed by BGK equation. The relaxation time is calculated by the relation $\tau=\mu /(\rho R T)$. The initial temperature $T_{0}$, pressure $P_{0}$ and density $\rho_{0}$ are the same in both parts of the slab. However, the temperature of the right side of the plate $T_{w}$ differs from the temperature of its left side $T_{0}$. As the result, the temperature, as well as the pressure, increases in the right part of the slab. Finally, the motion of the plate is induced by the difference of pressure at each side of the plate:

$$
m \frac{d u_{p}}{d t}=\left(P^{-}-P^{+}\right) S
$$

where $u_{p}$ is the velocity of the plate, $P^{-}$and $P^{+}$are the pressures at the left and right side of the plate, $m$ is the mass of the plate and $S$ is its surface. The experiment is represented Fig. 3.

The equilibrium position of the plate can be analytically calculated. Indeed, if we write the mass conservation and the gas equation of state for each part of the slab:

$$
\left\{\begin{array} { l } 
{ \rho _ { \text { left } } R T _ { 0 } = P _ { \text { equi } } } \\
{ \rho _ { \text { right } } R T _ { w } = P _ { \text { equi } } }
\end{array} \quad \text { and } \quad \left\{\begin{array}{l}
\rho_{0} L_{0}=\rho_{\text {left }}\left(L_{0}+x_{\text {equi }}\right) \\
\rho_{0} L_{0}=\rho_{\text {right }}\left(L_{0}-x_{\text {equi }}\right)
\end{array},\right.\right.
$$

we have:

$$
x_{\text {equi }}=L_{0} \frac{1-T_{w} / T_{0}}{1+T_{w} / T_{0}}, \quad \rho_{\text {left }}=\frac{P_{\text {equi }}}{R T_{0}}, \quad \rho_{\text {right }}=\frac{P_{\text {equi }}}{R T_{w}}, \quad \text { and } \quad P_{\text {equi }}=\frac{L_{0}}{L_{0}+x_{\text {equi }}} P_{0} .
$$


The experiment has been numerically studied. The initial temperature $T_{0}$, velocity $u_{0}$, pressure $P_{0}$ and the right temperature of the plate $T_{w}$ are respectively set to $270 \mathrm{~K}, 0 \mathrm{~m} \cdot \mathrm{s}^{-1}, 1 \mathrm{~Pa}$ and $330 \mathrm{~K}$. The total length of the plate is given by $2 L_{0}=2 m$. With these parameters, we get $x_{\text {equi }}=-0.1 \mathrm{~m}$ and the Knudsen number is $K n=5 \cdot 10^{-3}$. For this range of Knudsen numbers, the Navier-Stokes equations are still valid.

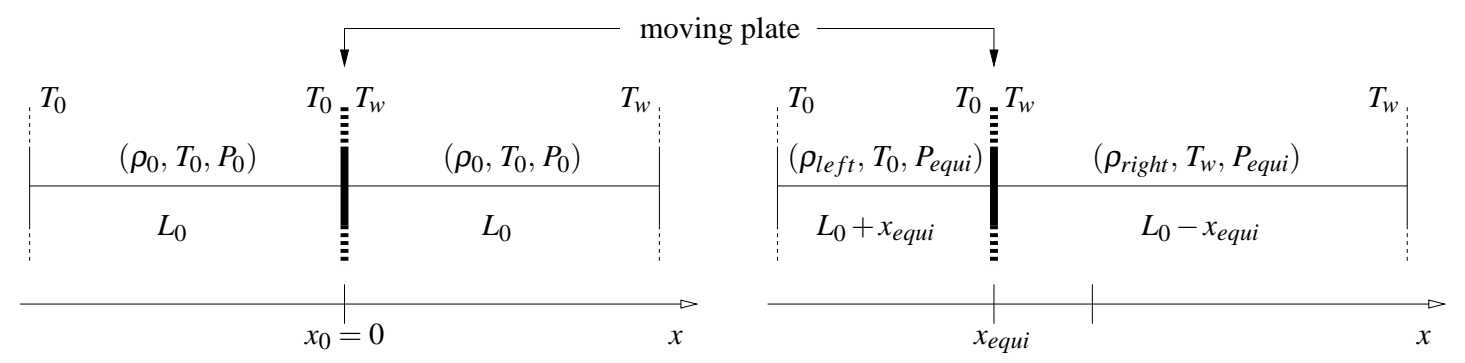

FIGURE 3. Actuator experiment at initial state (left) and equilibrium state (right).

First, the moving mesh method is applied to solve the BGK equation. The BGK equation is independently solved on left and right parts of the slab. For the left part of the slab, the diffuse boundary conditions at the fixed wall and at the moving plate are respectively implemented with $\left(T_{0}, u\right)=(270,0)$ and $\left(T_{0}, u\right)=\left(270, u_{p}^{n}\right)$. The diffuse boundary conditions for the right part of the slab are implemented in the same way, that is $\left(T_{w}, u\right)=\left(330, u_{p}^{n}\right)$ at the moving plate and $\left(T_{w}, u\right)=(330,0)$ at the fixed wall. The velocity and the position of the plate are computed with backward Euler schemes:

$$
\begin{aligned}
& u_{p}^{n+1}=u_{p}^{n}+\Delta t \times \frac{S}{m}\left(P_{l, N_{x}}^{n}-P_{r, 1}^{n}\right), \\
& x_{p}^{n+1}=x_{p}^{n}+\Delta t \times u_{p}^{n},
\end{aligned}
$$

where $P_{l, N_{x}}^{n}$ and $P_{r, 1}^{n}$ are the pressures in the last and first cell of the left and right part of the slab at time $t^{n}$. Velocity and position of the plate are plotted Fig. 4 . Notice that the equilibrium time is about $t \approx 100 \mathrm{~s}$. Indeed, the plate is oscillating around its equilibrium position until its velocity comes to zero. Moreover the equilibrium position is in agreement with relation (24).
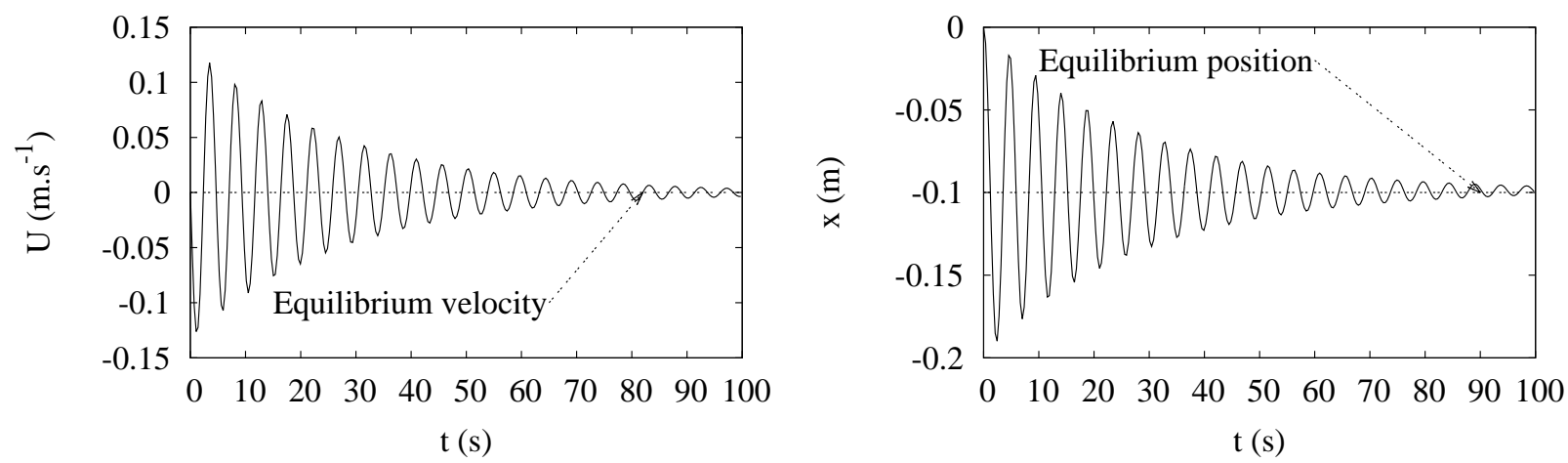

FIGURE 4. Left: velocity of the moving plate. Right: position of the moving plate

In a second step, BGK equation are discretized with the immersed boundary method [4]. With this approach the grid is unchanged during the whole computation. Then, every cell which center is located in a solid object is considered as solid. At last, the value of the distribution function in these solid cells is chosen in order to impose an appropriate value of $f$ at the solid interface. The results are in good agreement with the moving mesh method, see Fig. 5. This numerical result shows no difference between the two methods. But we can expect that the moving mesh method will lead to more accurate results that the immersed boundary method for more complex computations including among others $2 \mathrm{D}$ simulations. Indeed, unlike the immersed boundary method, the moving mesh method set the numerical flux to zero at the boundary, so that the total mass is preserved. 
Finally, it is assumed that the gas is also governed by Navier-Stokes equations. These one are written in Lagrangian coordinates and are solved by using the central scheme of Nessayhu-Tadmor [14]. Here again, the position of the plate, Fig. 5, is really closed to the one obtained with BGK equation.

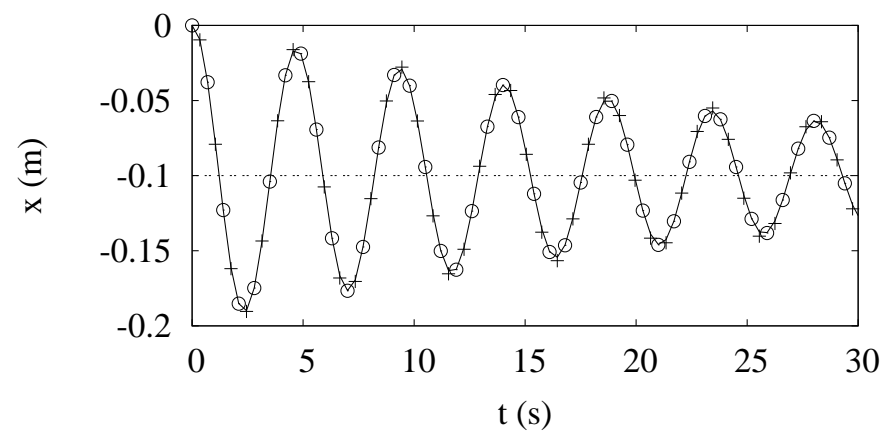

FIGURE 5. Position of the moving plate: BGK equation with moving mesh method, $\odot$ BGK equation with Immersed boundary method, + Navier stokes equations with a full Lagrangian scheme.

\section{CONCLUSION}

We proposed an extension of the moving mesh method, already used for the simulation of compressible viscous flows, to the simulation of the BGK model of gas dynamics. The method has been validated for one dimensional problems and we showed that the method accurately predicts the behaviour of the gas near the boundary. The extension of the method to higher dimensional problems is challenging because the recalculation of mesh seems difficult. However, several methods, such that ALE approaches [15], on unstructured grid have been proposed for the computation of compressible flow and our project is to use these methods for the computation of rarefied gas flows.

\section{REFERENCES}

1. C. Cercignani, The Boltzmann Equation and Its Applications, vol. 67 of Applied Mathematical Sciences, Springer-Verlag, 1988.

2. G. Karniadakis, A. Beskok, and N. Aluru, Microflows and Nanoflows: Fundamentals and Simulation, vol. 29 of Interdisciplinary applied mathematics, Springer, 2002.

3. C. Peskin, Annu. Rev. Fluid Mech. 14, 235-259 (1981).

4. G. Dechristé, and L. Mieussens, J. Phys.: Conf. Ser. 362 (2012).

5. T. Tang, Moving mesh methods for computational fluid dynamics, vol. 383 of contemporary Mathematics, AMS, 2005.

6. P. L. Bhatnagar, E. P. Grosss, and M. Krook, Phys. Rev. 94, 511-525 (1954).

7. G. A. Bird, Molecular Gas Dynamics and the Direct Simulation of Gas Flows, vol. 42 of Oxford Engineering Science Series, Oxford Science Publications, 1994.

8. S. Chapman, and T. G. Cowling, The mathematical theory of non-uniform gases, Cambridge University press, 1970.

9. L. Mieussens, Math. Mod. Meth. Appl. S. 10, 1121-1149 (2000).

10. R. J. LeVeque, Finite volume methods for hyperbolic problems, Cambridge University Press, 2002.

11. L. Pareschi, and G. Russo, J. Sci. Comp. 25, 129-155 (2005).

12. G. Russo, and F. Filbet, Kinetic and related models 2, 231-250 (2009).

13. E. Godlewski, and P.-A. Raviart, Numerical Approximation of Hyperbolic System of Conservation Laws, vol. 118 of Applied Mathematical Sciences, Springer, 1996.

14. H. Nessyahu, and E. Tadmor, J. Comput. Phys. 87, 408-463 (1990).

15. E. Kuhl, S. Hulshoff, and R. de Borst, Int. J. Numer. Meth. Engng 57, 117-142 (2003). 